and patients were identified for safe and early discharge with early community follow up. A total of 641 bed days were saved over the time period analysed.

Conclusion Implementation of a 7-day HALS team covering two acute hospital sites has significantly improved the quality of care provided to patients with alcohol-related disorders. In addition there has been a very positive impact on reducing length of stay and bed occupancy. Plans are in place to develop and audit the HALS team further and strengthen working relationships with community partners.

Disclosure of Interest None Declared.

\section{OC-039 IMPROVING QUALITY IN A NATIONAL INTESTINAL FAILURE UNIT: GREATER EFFICIENCY, IMPROVED ACCESS, REDUCED MORTALITY}

${ }^{1} \mathrm{E}$ Donaldson*, ${ }^{2} \mathrm{M}$ Taylor, ${ }^{2} \mathrm{~A}$ Abraham, ${ }^{2} \mathrm{G}$ Carlson, ${ }^{2} \mathrm{O}$ Fletcher, ${ }^{2} \mathrm{~V}$ Jacqui, ${ }^{2} \mathrm{~A}$ Teubner, ${ }^{2} S$ Lal. 'Gastroenterology/Quality Improvement, Salford Royal NHS Foundation Trust, Salford, UK; ${ }^{2}$ Intestinal Failure Unit, Salford Royal NHS Foundation Trust, Salford, UK

\subsection{6/gutjnl-2014-307263.39}

Introduction In 2010, there was a significant waiting list for admission to the Intestinal Failure Unit (IFU) at Salford, one of two current nationally-accredited centres. There had also been a steady increase in referrals to the IFU (89 patients in 2005; 152 patients in 2012) and the number of established patients requiring home parenteral nutrition (HPN) (135 patients in 2005; 206 patients in 2012). The impact of the long waiting list for these complex patients was that patient deaths occurred in those awaiting admission. Furthermore, the 'Strategic Framework for IF and HPN Services' in England had earlier highlighted the need for services to 'foster equity of access'. ${ }^{1}$ The SRFT IFU team therefore conducted a rigorous assessment of its processes in order to improve patient flow and access to the IFU. The primary aim was to reduce inpatient length of stay (LOS) by $10 \%$.

Methods We employed continuous improvement methodology, utilising the Model for Improvement and running sequential Plan-Do-Study-Act cycles. In addition to the key flow data, such as LOS and referral times, process data were collected, including time to intravenous feeding line insertion, time to radiology studies etc., when looking to streamline specific areas of practice. Data were analysed using statistical process control charts produced using QI Macros (KnowWare International, INC.). Statistically significant shifts were determined 'a priori' according to standard operating principals for special cause variation. ${ }^{2}$

Results Process improvements yielded a $20.8 \%$ reduction in average length of stay on the IFU from 55.7 to 44.1 days and a reduction of $70.7 \%$ in the average length of time spent on the waiting list for admission from 65.1 to 19.1 days. These changes were associated with concomitant reduction in 30-day readmission rates from 12.1 to $4.5 \%$ and early suggestions of reduced waiting list mortality. The number of inpatient deaths did not increase; indeed, there was a sustained increase in the number of complete discharge episodes between inpatient deaths (mean increase from 13 to 44).

Conclusion A quality improvement model is an effective means of enhancing the efficiency of a large National centre dealing with complex medical and surgical patients. Improvements in inpatient efficiency can reduce waiting times for admission, thus improving access and reducing waiting list mortality. The improvements in efficiency can be achieved without compromising patient safety.

\section{REFERENCES}

1 Strategic Framework for Intestinal Failure and Home Parenteral Nutrition Services for Adults in England 2008

2 Langley, G et al. 2009. The improvement guide: a practical approach to enhancing organizational performance. John Wiley \& Sons

Disclosure of Interest None Declared.

\section{OC-040 COMMUNITY-BASED ENDOSCOPY IN A CAR PARK. FANTASY OR REALITY?}

A Hitchen, B Kompo*, P Phillips, E Price, G Deans, NK Ahluwalia. Gastroenterology, Care UK, Manchester, UK

\subsection{6/gutjnl-2014-307263.40}

Introduction Owing to safety and quality concerns raised by BSG in 1990s, Community Based Endoscopy (CBE) rapidly declined. In 2014 CBE is back on the agenda with CCGs demanding a safe, high-quality, sub-tariff, 7 day endoscopy service close to the patient's home, at a time of the patient's choosing. We describe 5 years experience of a fully JAG accredited consultant-delivered completely mobile endoscopy service provided in shopping centre car parks throughout Greater Manchester (GM).

Methods In 2007, concept emerged out of a tender from 10 PCTs demanding safe and high quality endoscopy in the community. A linked-3-trailer unit with spacious waiting area for patients/relatives, consent-counselling, preparation, examination, decontamination and recovery areas. with its own water, electricity, waste disposal, e-communications and administration network was commissioned with integral office, staff kitchen/rest room and changing area. All staff undergo rigorous mandatory induction and regular updates, $\mathrm{CPD}$, audits as per JAG. Emergency scenarios are regularly rehearsed and audited. Full complement of ALS certified staff support consultants 8 am-8 pm, 7/7 [360] days in diagnostic UGI and LGI endoscopies [including polypectomies] and deliver 36 units/day. All patients undergo JAG-standard monitoring of pulse, $\mathrm{BP}, \mathrm{O}_{2}$ saturation, sedation and pain scores. All records are paperless, live and e-MAXIM andUNISOFT-based. All patients are contacted within $24 \mathrm{~h}$ by an experienced nurse to record any untoward incident. Patient and family feedback and regular meetings with GP allows total quality management in service delivery. Though no age limit, patients with IDDM, BMI $>40,>25$ st and ASA $\geq 3+$ are excluded. Quarterly audits are shared with commissioners. Unit relocates biweekly to 7 convenient locations with adjacent free parking.

Results Of 26599 (10539 UGI, 10583 Flexi-Sig, 5477 Colonoscopy) procedures, 1 in 3 patients opted for out-of-hours or weekend as first choice.

LGI- 93\% good-bowel prep, 91\% caecal intubation, 8.8\% adenoma detection, $97 \%$ polyp recovery and $100 \%$ Bx for diarrhoea. UGI- 98\% D2 intubation, 100\% 6 week repeat for GU, $84 \%$ unsedated. Biannual JAG-GRS compliant audits showed 0\% 30 day mortality, 0\% UGI SAE and $5(0.018 \%)$ unplanned hospital admissions 4 requiring surgery due to colon perforation [one post-flexi detected in the unit (diverticular disease), 1 from polypectomy, 2 diagnostic colonoscopy] and fifth due to hyponatremia (CitraFleet - now discontinued) requiring electrolyte correction.

Conclusion CBE is ready for prime time, just at the right time, as commissioners are now seeking more care in the community 